\title{
Acute Intravenous Tissue Plasminogen Activator Therapy does not Impact Community Discharge after Inpatient Rehabilitation
}

Nneka L Ifejika ${ }^{1,2 *}$, Farhaan Vahidy ${ }^{1}$, Linda A Aramburo-Maldonado ${ }^{2}$, Chunyan Cai ${ }^{3}$, Melvin R Sline ${ }^{1}$, James C Grotta ${ }^{4}$ and Sean I Savitz ${ }^{1}$

${ }^{1}$ Department of Neurology (N.L.I., F.V., L.A.A., M.R.S., S.I.S,), University of Texas Health Science, Center at Houston Medical School, Houston, Texas, USA

${ }^{2}$ Department of Physical Medicine and Rehabilitation (N.L.I.), University of Texas Health Science Center at Houston Medical School, Houston, Texas, USA

${ }^{3}$ Division of Clinical and Translational Sciences, Department of Internal Medicine (C.C.), University of Texas Health Science Center at Houston Medical School, Houston, Texas, USA

${ }^{4}$ Mischer Neuroscience Institute (J.C.G.), Memorial Hermann Hospital-Texas Medical Center, Houston, Texas, USA

\begin{abstract}
Background and purpose: Discharge status and acute re-hospitalization are used as indicators of stroke severity and recovery. Intravenous t-PA (tissue plasminogen activator) is one of two treatments shown to have a positive impact. Stroke rehabilitation patients are an important population who will end up integrated back into the community, institutionalized or hospitalized due to late stroke complications. We sought to determine factors contributing to post rehabilitation discharge and acute re-hospitalization, in particular, the impact of t-PA therapy.
\end{abstract}

Methods: Retrospective analysis of census data from ischemic stroke patients on the UTHealth Stroke/ Neurorehabilitation Services at Memorial Hermann Hospital - Texas Medical Center between Jan 2011 and Nov 2013, discharged to the Community, SNF (Skilled Nursing Facility) or AC (Acute Care). Demographics and NIHSS (National Institutes of Health Stroke Scale) were collected. Discharge FIM (Functional Independence Measure) was the reference standard. Genitourinary infections were a negative mediator in the multivariate regression.

Results: Of 346 patients, 274 returned to the community, 47 to SNF, and 25 to AC.

NIHSS and t-PA therapy: Median NIHSS values were 8 in the community group, 11 in SNF and 9.5 in AC.

$31.8 \%$ of patients received IV t-PA in the community group, $23.4 \%$ in SNF and $24 \%$ in AC. There were no statistically differences in community discharge rates.

Community vs. AC: One day increase in rehabilitation hospitalization correlated with $19 \%$ decreased odds of $A C$ readmission (OR 0.81; $P=0.001)$. One unit discharge FIM increase correlated with $13 \%$ decreased odds of $A C$ readmission (OR 0.87; $\mathrm{P}=0.003$ ).

Community vs. SNF: One year age increase correlated with $4 \%$ increased odds of SNF admission (OR 1.04; $\mathrm{P}=0.02)$.

Conclusions: Intense rehabilitation evidenced by FIM improvement and length of stay, impacts community discharge in mild to moderate stroke patients. t-PA had no effect. This study is limited by sample size, retrospective design and undetermined psychosocial factors.

Keywords: Stroke rehabilitation; Outcomes; Acute thrombolysis; Hospital re-admission; Skilled nursing facility; Functional independence measure

\section{Introduction}

Cerebrovascular disease is the leading cause of disability in the United States, costing an estimated $\$ 36.5$ billion annually [1]. To curb cost and decrease disability rates, iterations of outcomes research are of critical importance, particularly related to post-acute rehabilitation care. In 1995, the NINDS [National Institute of Neurologic Disorders and Stroke] and Stroke t-PA (tissue plasminogen activator) trial showed improved functional outcomes at three months in patients treated with t-PA compared to placebo [2]. However, the impact of t-PA on the rehabilitation process and acute care re-hospitalization during those three months has not been elucidated.

Early efforts to quantify the impact of intravenous thrombolysis on the need for inpatient rehabilitation in the acute stroke setting have been promising. In a 2011 study, acute stroke patients who received t-PA were found to have significant improvement in their NIHSS [National Institutes of Health Stroke Scale] values and increased likelihood of community discharge without an inpatient rehabilitation stay [3]. Unfortunately for the acute stroke patient who requires inpatient rehabilitation, the impact of intravenous thrombolysis on NIHSS score and eventual community discharge is unknown.
In comparison to Vascular Neurologists, rehabilitation professionals such as physiatrists, physical therapists, occupational therapists and speech and language pathologists use separate and distinct outcome measures to determine community discharge in stroke patients. Measures of rehabilitation success and quality include discharge status, rates of re-hospitalization and improvement in FIM (functional independence measure) score. Consisting of eighteen items in seven domains, the FIM score provides a uniform assessment of the severity of disability based on the level of independence [4,5]. During the rehabilitation hospitalization, FIM scores provide objective data that is

*Corresponding author: Nneka L Ifejika, Department of Neurology (N.L.I., F.V L.A.A., M.R.S., S.I.S,), University of Texas Health Science Center at Houston Medical School, Houston, Texas, USA, Tel: (713)500-7085; Fax:(713)500-0692 E-mail: Nneka.L.Ifejika@uth.tmc.edu

Received August 19, 2015; Accepted October 09, 2015; Published October 15 2015

Citation: Ifejika NL, Vahidy F, Aramburo-Maldonado LA, Cai C, Sline MR, et al (2015) Acute Intravenous Tissue Plasminogen Activator Therapy does not Impact Community Discharge after Inpatient Rehabilitation. Int J Neurorehabilitation 2: 183. doi:10.4172/2376-0281.1000183

Copyright: $\odot 2015$ Ifejika NL, et al. This is an open-access article distributed under the terms of the Creative Commons Attribution License, which permits unrestricted use, distribution, and reproduction in any medium, provided the original author and source are credited. 
used to track improvements and decompensations on a daily basis [6]; this data is used to facilitate discharge planning in those with high FIM scores and preparation for placement in a skilled nursing facility for those with low FIM scores [7].

FIMvalues in the inpatient rehabilitation setting can be detrimentally impacted by HAIs (healthcare associated infections) [8]. HAIs in the acute stroke patient have been associated with poor outcomes due to both prolonged hospitalization for antibiotic treatment and suboptimal participation in acute rehabilitation [8-10]. The most prevalent, SUTI (symptomatic urinary tract infection), accounts for over $21 \%$ of HAI incidence; studies have found that UTI [urinary tract infection] is one of the most frequent complications of stroke, with rates of 1 to $24 \%$ within the first month [11-13]. Sien Ng et al. [14] noted that higher discharge FIM score was associated the absence of HAI's. Furthermore, patients with medical complications during inpatient rehabilitation are at a higher risk for poorer functional outcomes at 1 year. Therefore, the identification and treatment of modifiable factors, such as SUTI, allow for optimal stroke rehabilitation $[14,15]$.

The primary outcome of acute thrombolysis trials has focused on a clinical endpoint of ninety day mRS (modified Rankin Scale) value [16]. However, patients and their families are interested in the events that occur during those three months that lead to the end result, namely, the process of rehabilitation care.

The purpose of this study is to determine whether intravenous thrombolysis with t-PA and the subsequent improvement in NIHSS can predict community discharge after inpatient rehabilitation. Discharge FIM value will serve as the reference standard for return to the community. Furthermore, we will evaluate the impact of SUTI as a healthcare associated infection on discharge FIM values.

\section{Subjects and Methods}

This is a CQI (continuous quality improvement) project initiated as a part of the Neurorehabilitation Subcommittee of our TJC (The Joint Commission) certified CSC (Comprehensive Stroke Center), MHHTMC (Memorial Hermann Hospital - Texas Medical Center). Quality improvement projects are integral to establishing safety guidelines, decreasing the costs of care and evaluating root-cause analyses. MHH-TMC is a tertiary care center with an in-house inpatient rehabilitation unit; all patients who meet admission criteria to inpatient Neurorehabilitation are accepted regardless of insurance type or ability to pay for services rendered. Therefore, our data set is characterized as census data, an official survey of all eligible participants.

For this project, we merged two prospectively collected quality assured databases. We performed a data query of the fields of interest from our university-based UT-Houston Medical School Stroke Registry, a collection of 200 variables collected prospectively on each patient admitted to the stroke service [17]. The registry was maintained by the SPOTRIAS (Specialized Programs of Translational Research in Acute Stroke) Data Core, a national network of 8 centers that perform early phase clinical projects, share data, and promote new approaches to therapy for acute stroke. Demographic information, SUTI on the Stroke service and NIHSS values were abstracted from the UT-Houston Stroke Registry. Cardiovascular Disease, Diabetes Mellitus, Hypertension, and History of Stroke did not show a significant impact on admission to inpatient rehabilitation in our previous studies $[15,18]$; instead, the patient's ability to tolerate three hours of therapy daily was used as the physical standard for inpatient rehabilitation admission [9]. Therefore, these medical comorbidities were excluded.
From the rehabilitation database, we collected length of stay, FIM score on admission and discharge, SUTI while on the Neurorehabilitation service and post-rehabilitation discharge disposition. The registries were matched by discharge date from the Stroke service and admission date to the Neurorehabilitation service, accounting for greater than $57 \%$ of the data. The remaining records required the use of a data abstractor, who manually matched cases.

Post Inpatient Rehabilitation Discharge Dispositions were defined as follows:

- Community - A private, community-based dwelling (a house, apartment, mobile home, etc.) that houses the patient, family or friends.

- SNF (Skilled Nursing Facility) - A setting that provides skilled services, including physical, occupational and speech therapy for an average of 1.5 hours daily. Care is ordered by a physician, a medical record is maintained and nursing services are present 24 hours a day.

- AC (Acute Care) - An acute medical/surgical care unit where patients receive treatment by a physician and their team.

Our primary objective was to determine whether t-PA therapy is associated with post-rehabilitation discharge disposition. We hypothesized that the presence of SUTI and/or advanced age would negatively impact the stroke rehabilitation hospital course, and ultimately decrease the likelihood of community discharge. The primary dependent variable was post-inpatient rehabilitation discharge disposition, which served as the proxy for functional status $[2,16]$.

\section{Statistical Methods}

All statistical analyses were performed using STATA 13 (College Station, Texas). Continuous variables were summarized as mean \pm standard deviation, or as median with interquartile range based on their distribution. Categorical variables were reported as frequencies and proportions. Univariate comparisons for demographic and clinical variables were completed between patients with community discharge and patients who were discharged to AC or SNF. The independent variables were NIHSS score, t-PA therapy, Age, FIM score at discharge and FIM Efficiency (FIM score at admission minus FIM score at discharge divided by rehabilitation length of stay). SUTI can impact community discharge after inpatient rehabilitation, it was included to evaluate for potential effects.

Two separate logistic regression models were fit to assess the factors associated with a favorable discharge disposition (community discharge vs. AC, and community discharge vs. SNF). Factors that were clinically relevant or statistically significant at $10 \%$ level in the univariate analysis were included in the multivariate model. Likelihood ratio test was performed to assess for significance of a parsimonious model vs. the full model. Regression diagnostics were performed on the final model along with testing for the fit of the model using Hosmer and Lemeshow goodness of fit test. Level of significance for all hypothesis testing was set at 0.05 .

We created a diagram that details t-PA treatment rates in the community, AC and SNF groups to address concerns regarding intergroup differences. We also analyzed the effects of t-PA therapy on median NIHSS values and admission FIM score.

\section{Results}

Between January 2011 and November 2013, we identified 346 
ischemic stroke patients admitted to both the UT Stroke Service and the UT Neurorehabilitation Service, who were subsequently discharged from Inpatient Rehabilitation (IR) to the community, SNF or AC.

\section{Effect of IV t-PA therapy and NIHSS value on post-} rehabilitation discharge disposition

Of 346 acute ischemic stroke patients, 274 returned to the community, 47 were transferred to a SNF, and 25 were readmitted to AC from the inpatient Neurorehabilitation service (Table 1 and 2). Our comprehensive stroke center treated $31.8 \%$ of ischemic stroke patients with intravenous t-PA in the community discharge group, $23.4 \%$ in the SNF discharge group and $24 \%$ in the AC readmission group. The proportion of patients treated with IV t-PA in the three disposition groups were not statistically different (Figure 1).

Median NIHSS values were 8 in the community discharge group, 9.5 in the AC readmission group and 11 in the SNF group. The median NIHSS scores of IV t-PA treated patients for the three disposition groups were not statistically different (Figure 1). IV t-PA treated patients who were discharged the community had significantly higher admission FIM values as compared to those who were discharged to SNF ( $\mathrm{p}=0.03$ ) (Figure 1).

\section{Univariate analysis: Community versus SNF - Post- rehabilitation discharge disposition}

Patients in the community discharge group were 9.7 years younger than the SNF group. For a one year increase in age, there were $5 \%$ increased odds of SNF admission (Table 1).

Admission FIM score was significantly higher in the community group (56.8) than the SNF group (44.4; $\mathrm{P}<0.0001)$. For a one unit increase in admission FIM, there were six percent decreased odds of SNF admission (Table 1).

Discharge FIM was significantly higher in the community group (78.2) versus the SNF group (61.2; $\mathrm{P}<0.0001)$. For a one unit increase in discharge FIM, there were seven percent decreased odds of SNF admission (Table 1). For a one unit increase in FIM efficiency, there were $51 \%$ decreased odds of SNF admission (Table 1).

\begin{tabular}{|c|c|c|c|c|}
\hline & $\begin{array}{c}\text { Community } \\
n=274(79.2 \%)\end{array}$ & $\begin{array}{c}\text { SNF } \\
\mathrm{n}=47(13.6 \%)\end{array}$ & OR $(95 \% \mathrm{Cl})$ & $P$ value \\
\hline Age, mean (SD) & $58.9(13.8)$ & $68.2(12.2)$ & $1.05(1.03-1.09)$ & $<0.001$ \\
\hline Gender, \% male & $155(56.6 \%)$ & $28(59.6)$ & $1.13(0.60-2.12)$ & 0.7 \\
\hline \multicolumn{5}{|l|}{ Ethnicity, n (\%) } \\
\hline African-American & $112(40.9 \%)$ & $13(27.6 \%)$ & $0.76(0.53-1.10)$ & 0.15 \\
\hline Hispanic & $46(16.8 \%)$ & $7(14.9 \%)$ & & \\
\hline Other Ethnicity & $21(7.7 \%)$ & $3(6.4 \%)$ & & \\
\hline Caucasian & $95(34.6 \%)$ & $24(51.1 \%)$ & & \\
\hline NIHSS, median (IQR) & $8(4-13)$ & $11(6-15)$ & $1.02(0.98-1.07)$ & 0.24 \\
\hline t-PA therapy, n (\%) & $87(31.8 \%)$ & $11(23.4 \%)$ & $0.65(0.32-1.34)$ & 0.25 \\
\hline Acute Stroke Length of Stay in Days, mean (SD) & $5.32(2.82)$ & $5.25(2.06)$ & $0.99(0.87-1.13)$ & 0.88 \\
\hline Neurorehabilitation Length of Stay in Days, mean (SD) & $14.11(6.38)$ & $15.81(5.78)$ & $1.04(0.99-1.09)$ & 0.09 \\
\hline UTI on Stroke service, $\mathrm{n}(\%)$ & $17(6.2 \%)$ & $2(4.3 \%)$ & $0.67(0.15-3.0)$ & 0.60 \\
\hline UTI on Neurorehabilitation service, $\mathrm{n}(\%)$ & $93(33.9 \%)$ & $17(36.2 \%)$ & $1.09(0.57-2.09)$ & 0.78 \\
\hline Admission Functional Independence Measure Score, mean (SD) & $56.8(14.98)$ & $44.4(12.24)$ & $0.94(0.92-0.96)$ & $<0.0001$ \\
\hline Discharge Functional Independence Measure Score, mean (SD) & $78.2(14.56)$ & $61.2(15.89)$ & $0.93(0.91-0.95)$ & $<0.0001$ \\
\hline FIM Efficiency (FIM change / Rehab Length of Stay), mean (SD) & $1.77(1.35)$ & $1.13(0.80)$ & $0.49(0.33-0.74)$ & 0.001 \\
\hline
\end{tabular}

Table 1: Demographic Information by Post-Rehabilitation Discharge Disposition - Community versus Skilled Nursing Facility (SNF).

\begin{tabular}{|c|c|c|c|c|}
\hline & $\begin{array}{c}\text { Community } \\
n=274(79.2 \%)\end{array}$ & $\begin{array}{l}\text { Acute Care } \\
n=25(7.2 \%)\end{array}$ & $\begin{array}{c}\text { OR } \\
(95 \% \mathrm{Cl})\end{array}$ & $P$ value \\
\hline Age, mean (SD) & $58.9(13.8)$ & $65.6(11.5)$ & $1.02(0.99-1.05)$ & 0.19 \\
\hline Gender, \% male & $155(56.6 \%)$ & $18(72 \%)$ & $1.97(0.80-4.88)$ & 0.14 \\
\hline \multicolumn{5}{|l|}{ Ethnicity, n (\%) } \\
\hline African-American & $112(40.9 \%)$ & $4(16 \%)$ & $0.89(0.56-1.41)$ & 0.62 \\
\hline Hispanic & $46(16.8 \%)$ & $6(24 \%)$ & & \\
\hline Other Ethnicity & $21(7.7 \%)$ & $2(8 \%)$ & & \\
\hline Caucasian & $95(34.6 \%)$ & $13(52 \%)$ & & \\
\hline NIHSS, median (IQR) & $8(4-13)$ & $9.5(6-16)$ & $1.0(0.95-1.07)$ & 0.17 \\
\hline t-PA therapy, n (\%) & $87(31.8 \%)$ & $6(24 \%)$ & $0.68(0.26-1.75)$ & 0.42 \\
\hline Acute Stroke Length of Stay in Days, mean (SD) & $5.32(2.82)$ & $5.52(2.80)$ & $1.02(0.87-1.21)$ & 0.78 \\
\hline Neurorehabilitation Length of Stay in Days, mean (SD) & $14.11(6.38)$ & $7.52(6.42)$ & $0.79(0.71-0.87)$ & $<0.0001$ \\
\hline UTI on Stroke service, n (\%) & $17(6.2 \%)$ & $1(4 \%)$ & $0.63(0.08-4.94)$ & 0.66 \\
\hline UTI on Neurorehabilitation service, n (\%) & $93(33.9 \%)$ & $13(52 \%)$ & $2.10(0.92-4.78)$ & 0.08 \\
\hline Admission Functional Independence Measure Score, mean (SD) & $56.8(14.98)$ & $45.9(13.78)$ & $0.95(0.92-0.98)$ & 0.001 \\
\hline Discharge Functional Independence Measure Score, mean (SD) & $78.2(14.56)$ & $49.1(16.54)$ & $0.90(0.87-0.93)$ & $<0.0001$ \\
\hline FIM Efficiency (FIM change / Rehab Length of Stay), mean (SD) & $1.77(1.35)$ & $0.66(1.65)$ & $0.33(0.20-0.54$ & $<0.0001$ \\
\hline
\end{tabular}

Table 2: Demographic information by post-rehabilitation discharge disposition - Community versus acute care. 


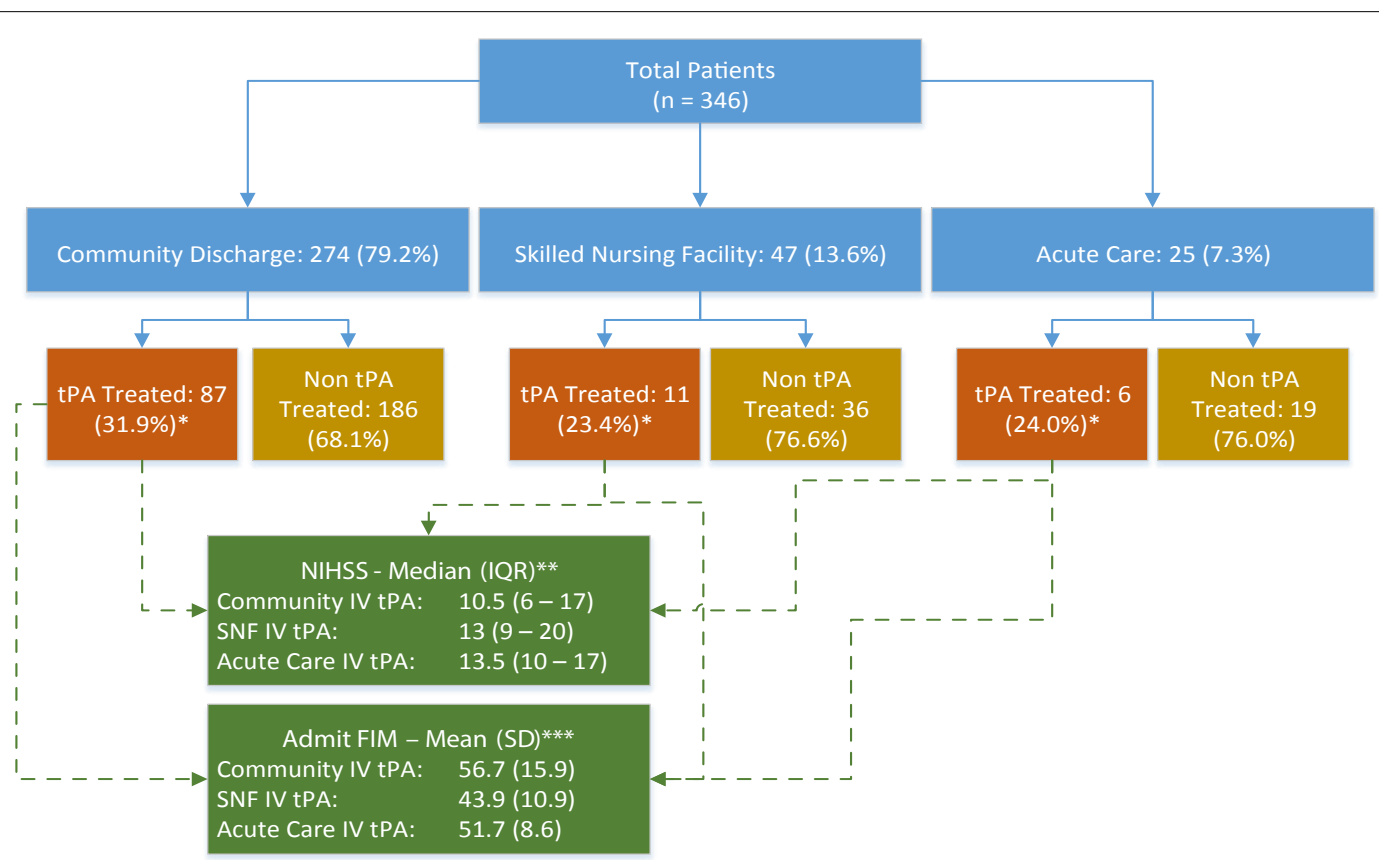

Figure 1: Median NIHSS (National Institutes of Health Stroke Scale) and Admission FIM (Functional Independence Measure) for Rehabilitation Patients who Received Intravenous t-PA (Tissue Plasminogen Activator) Therapy. *Proportion of patients treated with IV tPA in three disposition groups are not statistically different. **The median NIHSS scores of IV tPA treated patients for the three disposition groups are not statistically different. ${ }^{* *}$ IV tPA treated patients who were discharged the community have significantly higher admit FIM as compared to those who went to SNF $(p=0.03)$

\section{Univariate analysis: Community versus AC - Post- rehabilitation discharge disposition}

Patients who returned to AC had a shortened length of rehabilitation stay by 6.52 days compared to the community discharge group $(\mathrm{P}<0.0001)$. For a one day increase in rehabilitation length of stay, there were 21 percent decreased odds of AC readmission (Table 2).

Admission FIM score was significantly higher in the community discharge group (56.8) than the SNF group (45.9; $\mathrm{P}=0.001)$. For a one unit increase in admission FIM, there were six percent decreased odds of SNF admission (Table 2). Discharge FIM was significantly higher in the community group (78.2) versus the SNF group $(61.2 ; \mathrm{P}<0.0001)$. For a one unit increase in admission FIM, there were five percent decreased odds of $\mathrm{AC}$ readmission (Table 2).

Discharge FIM in the AC group was significantly lower (49.1) than discharge FIM in the community group $(78.2, \mathrm{P}<0.0001)$. For a one unit increase in FIM efficiency, there were $67 \%$ decreased odds of AC readmission (Table 2 ).

\section{Multivariate logistic regression}

For the disposition Community versus SNF, we included Age, Neurorehabilitation Length of Stay, Admission FIM, Discharge FIM and FIM Efficiency in the multivariate analysis. For a one year age increase, there were $4 \%$ increased odds of SNF admission (OR 1.04; $\mathrm{P}=0.02$ ). Rehab Length of Stay, Admission FIM, Discharge FIM or FIM efficiency were not significant (Table 3 ).

For the disposition Community versus AC, we included UTI on Neurorehabilitation, Neurorehabilitation Length of Stay, Admission FIM, Discharge FIM and FIM Efficiency in the multivariate analysis. For a one day increase in the Neurorehabilitation length of stay, there were 19 percent decreased odds of $\mathrm{AC}$ readmission ( $\mathrm{OR} 0.81 ; \mathrm{P}=0.001$ ). For every one unit increase in FIM score on discharge, there were 13

\begin{tabular}{|l|c|c|c|}
\hline Variable & Odds Ratio & $\mathbf{9 5 \%} \mathbf{~ C l}$ & P value \\
\hline Age & 1.04 & $1.01-1.08$ & 0.002 \\
\hline Neurorehabilitation Length of Stay & 0.97 & $0.87-1.06$ & 0.50 \\
\hline Admission FIM & 0.97 & $0.90-1.05$ & 0.47 \\
\hline Discharge FIM & 0.96 & $0.89-1.04$ & 0.33 \\
\hline FIM Efficiency & 0.76 & $0.29-2.02$ & 0.59 \\
\hline
\end{tabular}

Table 3: Multivariate logistic regression - Community versus SNF - Postrehabilitation discharge disposition.

\begin{tabular}{|l|c|c|c|}
\hline Variable & Odds Ratio & $95 \% \mathrm{Cl}$ & $\mathrm{P}$ value \\
\hline UTI on Neurorehabilitation Service & 1.36 & $0.41-4.51$ & 0.61 \\
\hline Neurorehabilitation Length of Stay & 0.81 & $0.72-0.92$ & 0.001 \\
\hline Admission FIM & 1.05 & $0.95-1.17$ & 0.30 \\
\hline Discharge FIM & 0.87 & $0.80-0.95$ & 0.003 \\
\hline FIM Efficiency & 0.82 & $0.40-1.70$ & 0.60 \\
\hline
\end{tabular}

Table 4: Multivariate logistic regression - Community versus acute care - Postrehabilitation discharge disposition.

percent decreased odds of AC readmission (OR 0.87; $\mathrm{P}=0.003$ ). UTI on Neurorehabilitation, Admission FIM and FIM efficiency were not significant (Table 4).

\section{Discussion}

This study provides new information on the impact of acute thrombolysis for ischemic stroke during inpatient rehabilitation. The administration of $\mathrm{t}$-PA therapy within 3 hours of symptom onset has been shown to increase community discharge rates after the acute hospitalization [3]. However, the five day duration of the acute stroke stay provides limited data on rehabilitation expectations. This study extends out the time window, establishing the impact of t-PA therapy on rehabilitation outcomes during the first 2.5 weeks post-stroke, a time of significant adjustment, anxiety and stress among stroke patients and their families. 
By analyzing all ischemic stroke patients who received rehabilitation care, we have created "census data", an official enumeration of the population without the influence of health insurance, a barrier that can block access to inpatient rehabilitation services.

Although IV t-PA therapy rates averaged $30 \%$ across the community, SNF and AC groups, the administration of t-PA therapy had no impact on community discharge. NIHSS values varied between the community, SNF and AC groups; however, there were no statistically significant differences. These findings reinforce the importance of inpatient rehabilitation and highlight a new finding - three hours of daily inpatient therapy can serve as an equalizer among patients with mild to moderate stroke who did not receive intravenous thrombolysis.

A secondary goal of stroke rehabilitation is to facilitate an appropriate change in FIM to improve a stroke survivor's functional mobility. This improvement in functional mobility leads to a decreased risk of debility related complications (falls, pressure ulcers, deep venous thrombosis). Therefore, the lower admission FIM scores in the t-PA treated group who were discharged to SNF are a function of preparing these patients for another level of post-acute care.

The diagnosis of SUTI had no significant impact on AC readmission rates from inpatient rehabilitation. This new information contrasts with AC studies, where the presence of SUTI was predictive of rehabilitation care in a SNF, a level of care suggestive of lower functional status and worse outcomes $[12,15]$. The obviated impact of UTI on community discharge is due to the standard protocol of collecting a urine sample within 24 hours of rehabilitation admission. Early detection of genitourinary infections facilitates rapid treatment, decreasing urosepsis rates.

Forty percent of stroke survivors experience moderate to severe neurologic impairments, requiring specialized rehabilitation care [19]. Since peak neurologic recovery occurs within the first three months of a cerebrovascular event [20], the provision of an evidencebased rehabilitation pathway throughout those three months is of critical importance. The presence of Neurorehabilitation services in comprehensive stroke centers provides continuity of care from Vascular Neurologists and Physiatrists as leaders of the multidisciplinary team - leading to a systematic and integrated assessment of neurologic improvement.

Large numbers of stroke survivors benefit from inpatient rehabilitation at centers that provide the full range of acute services, such as physical and occupational therapy, speech and language pathology, neuropsychology rehabilitation nursing, case management and social work [21]. Unfortunately, there are financial barriers to admission in a number of Neurorehabilitation centers. Lack of insurance or underinsurance can preclude inpatient rehabilitation, and in some cases, stroke survivors return home with only minimal caregiver services. This study was conducted at a tertiary care center with an in-hospital inpatient rehabilitation facility; therefore, all patients who meet criteria for admission to inpatient rehabilitation were accepted.

We cannot comment on the provision of caregiver services - if patient needs supervision for safety, has cognitive impairments or physical limitations, the identification of a caregiver is recommended before returning home. As an alternative to home, these patients may be admitted to an SNF or a personal care home, particularly in patients with advanced age, until a caregiver becomes available. This may account for our findings of skilled nursing facility placement rates of 13.6 percent, compared to an average of ten percent [22], as well as a four percent increased odds of SNF admission for a one year increase in age.
This analysis is retrospective; we endeavoured to stratify the data for age, stroke severity and known risk factors that suggest lower FIM scores and worse outcomes, such as SUTI. Our study did not define SUTI based on the Centers for Disease Control and Prevention guidelines. The reliability of using patient report of dysuria and suprapubic tenderness is attenuated, because many stroke patients cannot express symptoms due to aphasia or to the presence of indwelling urinary catheters.

\section{Summary/Conclusions}

Intense and sustained inpatient rehabilitation enhances the poststroke recovery process, particularly in patients who did not receive IV $\mathrm{t}$-PA. Acute ischemic stroke patients who received acute intravenous thrombolysis with t-PA had no difference in post-rehabilitation community discharge. All patients had mild to moderate strokes and were able to tolerate three hours of daily physical, occupational and speech therapy. Unlike the acute stroke hospitalization, symptomatic urinary tract infection had no impact on community discharge. A better understanding of the scientific process of Neurorehabilitation care during the first 3 months post-stroke can further improve the impact of this specialty.

\section{Acknowledgement}

We would like to thank the UTHealth Stroke and Neurorehabilitation Teams at Memorial Hermann Hospital - Texas Medical Center for their excellent patient care.

\section{Conflicts of Interest}

Dr. Ifejika is the Director of Neurorehabilitation at Memorial Hermann Hospital - Texas Medical Center.

\section{Sources of Funding}

Dr. Ifejika's and Dr. Cai's work is supported by the Center for Clinical and Translational Sciences at the University of Texas Health Science Center at Houston Medical School, which is funded by NIH/NCATS Clinical and Translational Award UL1 TR000371 and KL2 TR000370. The content is solely the responsibility of the authors and does not necessarily represent the official views of the National Center for Research Resources or the National Institutes of Health.

Dr. Ifejika's preliminary work was supported by the NIH/NINDS Diversity Supplement to P50 NS 044227, the University of Texas, Specialized Program of Translational Research in Acute Stroke (SPOTRIAS).

\section{References}

1. Go AS, Mozaffarian D, Roger VL, Benjamin EJ, Berry JD, et al. (2014) Heart disease and stroke Statistics-2014 update: A report from the American Heart Association. Circulation 28

2. Tissue plasminogen activator for acute ischemic stroke. The National Institute of Neurological Disorders and Stroke rt-PA Stroke Study Group. N Engl J Med 333: $1581-1587$

3. Ifejika-Jones NL, Harun N, Mohammed-Rajput NA, Noser EA, Grotta JC (2011) Thrombolysis with intravenous tissue plasminogen activator predicts a favorable discharge disposition in patients with acute ischemic stroke. Stroke 42: 700-704.

4. Lin JH, Hsiao SF, Chang CM, Huang MH, Liu CK, et al. (2000) Factors influencing functional independence outcome in stroke patients after rehabilitation. Kaohsiung J Med Sci 16: 351-359.

5. http://www.dementia-assessment.com.au/symptoms/FIM_manual.pdf

6. Chumney D, Nollinger K, Shesko K, Skop K, Spencer M, et al. (2010) Ability of Functional Independence Measure to accurately predict functional outcome of stroke-specific population: Systematic review. J Rehabil Res Dev 47: 17-29.

7. Denti L, Agosti M, Franceschini M (2008) Outcome predictors of rehabilitation for first stroke in the elderly. Eur J Phys Rehabil Med 44: 3-11.

8. Bottemiller KL, Bieber PL, Basford JR, Harris M (2006) FIM score, FIM efficiency, and discharge disposition following inpatient stroke rehabilitation. Rehabil Nurs 31: 22-25.

9. https://www.cms.gov/Outreach-and-Education/Medicare-Learning-NetworkMLN/MLNProducts/downloads/InpatRehabPaymtfctsht09-508.pdf 
Citation: Ifejika NL, Vahidy F, Aramburo-Maldonado LA, Cai C, Sline MR, et al. (2015) Acute Intravenous Tissue Plasminogen Activator Therapy does not Impact Community Discharge after Inpatient Rehabilitation. Int J Neurorehabilitation 2: 183. doi:10.4172/2376-0281.1000183

10. Johnsen SP, Svendsen ML, Ingeman A (2012) Infection in Patients with Acute Stroke. The Open Infectious Diseases Journal M3:40-45.

11. Oldenbeuving AW, de Kort PL, Jansen BP, Roks G, Kappelle LJ (2007) Delirium in acute stroke: a review. Int J Stroke 2: 270-275.

12. George AJ, Boehme AK, Siegler JE, Monlezun D, Fowler BD, et al. (2013) Hospital-Acquired Infection Underlies Poor Functional Outcome in Patients with Prolonged Length of Stay. ISRN Stroke 2013.

13. Davenport RJ, Dennis MS, Wellwood I, Warlow CP (1996) Complications after acute stroke. Stroke 27: 415-420.

14. Sien Ng Y, Astrid S, De Silva DA, Tan ML, Tan YL, et al. (2013) Functiona Outcomes after Inpatient Rehabilitation in a Prospective Stroke Cohort. Proceedings of Singapore Healthcare 22: 175-182.

15. Ifejika-Jones NL, Peng H, Noser EA, Francisco GE, Grotta JC (2013) Hospitalacquired symptomatic urinary tract infection in patients admitted to an academic stroke center affects discharge disposition. PM and R 5: 9-15.

16. Marshall RS (2015) Progress in Intravenous Thrombolytic Therapy for Acute Stroke. JAMA Neurol 72: 928-934.
17. Rahbar MH, Gonzales NR, Ardjomand-Hessabi M, Tahanan A, Sline MR, et al. (2013) The University of Texas Houston Stroke Registry (UTHSR): Implementation of enhanced data quality assurance procedures improves data quality. BMC Neurol 13: 61

18. Ifejika-Jones NL, Arun N, Peng H, Elizabeth A, Grotta JC, et al. (2012) The interaction of aspiration pneumonia with demographic and cerebrovascular disease risk factors is predictive of discharge level of care in acute stroke patient. Am J Phys Med Rehabil 91: 141-147.

19. Griffin LJ, Hickey JV (2013) Considerations and strategies for educating stroke patients with neurological deficits. J Nurs Educ Pract 3:125-137.

20. Nudo RJ (2003) Functional and structural plasticity in motor cortex: Implications for stroke recovery. Phys Med Rehabil Clin N Am 14: S57-76.

21. Bagherpour R, Dykstra DD, Barrett AM, Luft AR, Divani AA (2014) A Comprehensive Neurorehabilitation Program Should be an Integral Part of a Comprehensive Stroke Center. Front Neurol 5: 57.

22. Flick CL (1999) Stroke rehabilitation. Stroke outcome and psychosocia consequences. Arch Phys Med Rehabil 80: S21-S26. 